\title{
Adrenomyeloneuropathy as a cause of primary adrenal insufficiency and spastic paraparesis
}

\author{
Monika Spurek, Regina Taylor-Gjevre, Stan Van Uum, Hasnain M. Khandwala
}

Abstract

ADRENOMYELONEUROPATHY IS A VARIENT OF ADRENOLEUKODYSTROPHY, both of which are rare inherited disorders of peroxisomes characterized by the accumulation of very-long-chain fatty acids in plasma, the central and peripheral nervous systems, adrenal glands and testes, which leads to dysfunction of these organs and systems. In this article, we describe an illustrative case of adrenomyeloneuropathy and discuss the clinical presentation, diagnosis and management of the 2 disorders.

CMAJ 2004;171(9):1073-7

\section{Illustrative case}

A 35-year-old man with a 2-year history of bilateral, progressive weakness and spasticity in his lower extremities that had been diagnosed as idiopathic spastic paraparesis was evaluated after a syncopal episode. He also reported a 2- to 3-month history of abdominal pain, occasional vomiting and poor appetite before presentation. He had erectile dysfunction and overflow incontinence requiring intermittent self-catheterization. He did not describe changes in his libido or hair growth. His past medical history was remarkable for the absence of any trauma to the spine or autoimmune diseases. He denied the use of tobacco, alcohol and illicit drugs and had not travelled outside of his province. He had no family history of neurological or autoimmune disorders.

A physical examination was significant for a supine blood pressure of $90 / 60 \mathrm{~mm} \mathrm{Hg}$ and a pulse of 100 beats/min. The patient was afebrile. His skin was tanned, and he had pigmentation of the buccal mucosa. The respiratory, cardiovascular and abdominal examinations were unremarkable. Neurological examination revealed that the muscle bulk in his lower extremities was diminished, tone was increased and the power was reduced (3/5). Reflexes were brisk in the lower limbs, with evidence of ankle clonus. Sensation to all modalities was impaired in both lower limbs. His upper extremities appeared normal. There were no gross mental status changes or evidence of dementia, although detailed neuropsychological testing was not performed.

Laboratory investigations revealed a sodium level of 126 [normally $135-146$ ] mmol/L and elevated levels of po- tassium (6.6 [normally $3.5-5.1] \mathrm{mmol} / \mathrm{L})$, urea (19.6 [normally $3.7-7.0] \mathrm{mmol} / \mathrm{L}$ ) and creatinine (150 [normally 45-125] $\mu \mathrm{mol} / \mathrm{L})$. The complete blood cell count was normal. Given the presence of buccal pigmentation, hypotension, hyponatremia and hyperkalemia, a diagnosis of primary adrenal insufficiency was considered; the morning cortisol level of 60 (normally 130-640) nmol/L and adrenocorticotropic hormone (ACTH) level of $>278$ (normally $\leq 11) \mathrm{pmol} / \mathrm{L}$ subsequently confirmed the diagnosis. The serum testosterone level was normal.

The patient was hydrated intravenously and received glucocorticoid and mineralocorticoid replacement, and his blood pressure and electrolyte levels returned to normal. An MRI of the brain showed mild ventriculomegaly and a $1-\mathrm{cm}$ area of increased signal (on T2 and FLAIR images) adjacent to the occipital horn of the right lateral ventricle and a smaller, similar lesion adjacent to the anterior horn of the left lateral ventricle. The spine was normal in appearance. Results of a lumbar puncture were normal, and nerve conduction studies were suggestive of acquired demyelinating polyneuropathy.

Of the potential causes of primary adrenal insufficiency (Table 1), adrenomyeloneuropathy (AMN) is the only one that is associated with progressive spastic paraparesis. Verylong-chain fatty acids (VLCFAs) were measured in the plasma. $\mathrm{C}_{26: 0}$ levels were 2.33 (normally < 1.46) $\mu \mathrm{mol} / \mathrm{L}$, the $\mathrm{C}_{26: 0} / \mathrm{C}_{22: 0}$ ratio was 0.0567 (normally $\left.<0.035\right) \mu \mathrm{mol} / \mathrm{L}$, and

Table 1: Causes of primary adrenal insufficiency

\begin{tabular}{lc}
\hline Cause & Prevalence, \% \\
\hline Autoimmune disorder & 70 \\
Tuberculosis & 20 \\
Other & 10 \\
Fungal infection & \\
Adrenal hemorrhage & \\
AIDS & \\
Metastases & \\
ALD or AMN & \\
Congenital adrenal hypoplasia & \\
Drug use (ketoconazole, mitotane, metyrapone, \\
aminoglutethemide)
\end{tabular}

Note: $\mathrm{ALD}=$ adrenoleukodystrophy, $\mathrm{AMN}$ = adrenomyeloneuropathy. 
the $\mathrm{C}_{24: 0} / \mathrm{C}_{22: 0}$ ratio was 1.5 (normally < 1.10$) \mu \mathrm{mol} / \mathrm{L}$. Given these biochemical findings and the clinical presentation, the diagnosis of AMN was established. The patient underwent genetic studies, which revealed a single sequence variation in the $\mathrm{X}$-linked adrenoleukodystrophy (X-ALD) gene. At nucleotide 1587, the normal "C" nucleotide was replaced by a "T" nucleotide, which resulted in the substitution of tryptophan for arginine at amino acid 401 and was consistent with a known $\mathrm{X}$-linked AMN mutation. The patient was enrolled in a program of intensive physiotherapy and rehabilitation in addition to supplemental hydrocortisone and fludrocortisone therapy; no specific treatment of the underlying AMN was initiated. Screening of the patient's family members was also recommended. On a recent 6-month follow-up visit, the patient was clinically euadrenal and had stable neurological findings.

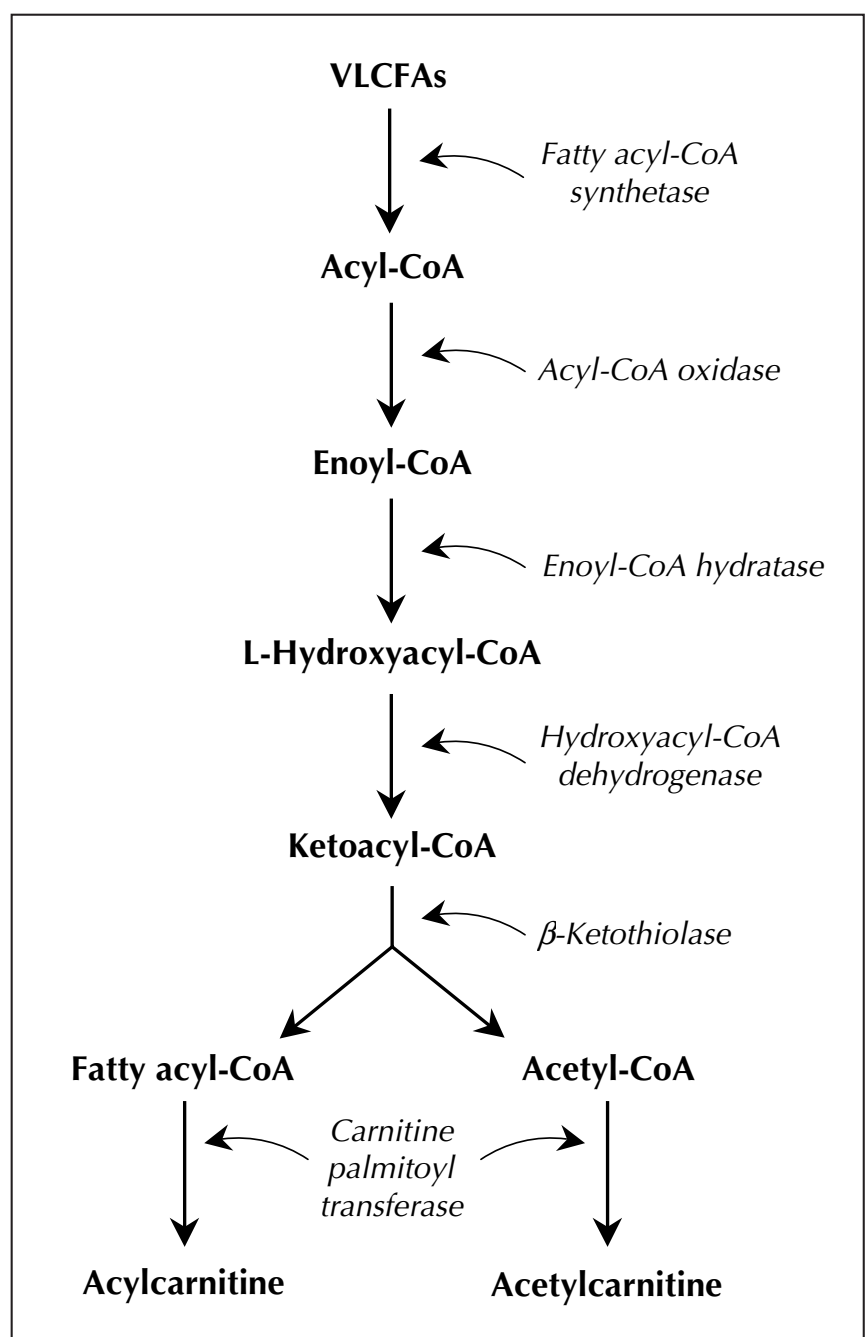

Fig. 1: The catabolism of very-long-chain fatty acids (VLCFAs) in peroxisomes. People with adrenoleukodystrophy or adrenomyeloneuropathy do not produce acylcoenzyme A (acyl-CoA) synthetase, which breaks down VLCFAs to their CoA esters in the first step in their oxidation.

\section{Pathogenesis}

AMN is a varient of adrenoleukodystrophy (ALD), both of which are rare sex-linked disorders transmitted on the $\mathrm{X}$ chromosome. Both conditions are characterized by progressive neurological symptoms and dysfunction of the adrenal glands and testes. ${ }^{1}$ Both disorders can cause adrenal insufficiency and hypogonadism in addition to neurological symptoms. In general, ALD tends to present at an earlier age and primarily involves the brain, whereas AMN occurs later and involves the spinal cord. The frequency of X-ALD or X-AMN in the US male population is $1: 42000$. The estimated combined frequency of $\mathrm{X}$ ALD and X-AMN in hemizygotes and heterozygotes is $1: 16000 .^{2}$

Both are disorders of the peroxisomes, which are intracellular organelles responsible for a variety of biochemical reactions, the most important being the oxidation of VLCFAs, biosynthesis of bile acids and glycoxylate detoxification. ${ }^{3}$ The oxidation of VLCFAs (hexacosanoic acid $\left[\mathrm{C}_{26: 0}\right]$, pentacosanoic acid $\left[\mathrm{C}_{25: 0}\right]$ and tetrocosanoic acid $\left.\left[\mathrm{C}_{24: 0}\right]\right)$ in the peroxisomes involves several steps that are catalyzed by different enzymes (Fig. 1). People with ALD or AMN do not produce fatty acylcoenzyme A (acyl-CoA) synthetase, which breaks down VLCFAs in the first step in their oxidation (Fig. 1). ${ }^{4}$ VLCFAs therefore accumulate and form cytoplasmic inclusions, which leads to progressive dysfunction in the nervous system, adrenal glands and testes, all of which are sites of VLCFA metabolism. In cerebral variants of $\mathrm{ALD}$, an inflammatory response is also seen, which is thought to damage myelin. Pathologically, these disorders are characterized by the presence of lamellar cytoplasmic inclusions consisting of cholesterol esterified with VLCFAs (Fig. 2). ${ }^{5}$ Siemerling and Creutzfeldt ${ }^{6}$ described the first pos- $^{-}$ sible case of ALD 7 decades ago, and in 1976, AMN was first reported to be a variant of ALD. ${ }^{7,8}$ Both disorders are transmitted in an $\mathrm{X}$-linked fashion: the gene is located on the long arm of the $\mathrm{X}$ chromosome $\left(\mathrm{Xq}_{\mathrm{q}} 2\right)^{9}$ and encodes a peroxisomal membrane protein belonging to the adenosine triphosphate-binding cassette superfamily of transporter proteins. A variety of missense, nonsense mutations, single amino acid deletions, frameshifts and splice acceptor-site defects have been identified. ${ }^{10}$

\section{Clinical presentation}

There is significant clinical heterogeneity in ALD, and $\mathrm{AMN}$ is one of several variants (Table 2). ${ }^{11}$ The 2 most frequent phenotypes are childhood cerebral ALD and AMN, which account for about $45 \%$ and $25 \%$ of all cases respectively. Recent evidence, however, suggests that AMN may be the more common form..$^{12}$ Classic ALD is characterized by rapidly progressive cerebral demyelination leading to spastic tetraparesis, dementia, seizures, and visual and auditory dysfunction. The adolescent and adult cerebral forms of ALD resemble the classic form but are 
later in onset. Neurological symptoms in AMN generally occur in the third and fourth decade and are characterized primarily by involvement of long ascending and descending tracts of the spinal cord and peripheral neuropathy due to demyelination, which leads to spastic paraparesis and urinary and erectile dysfunction. Although patients with AMN generally do not have clinically significant cerebral involvement, up to $50 \%$ of males with AMN have evidence of cerebral demyelination on MRI. AMN is generally considered to be a milder variant of ALD with slower progression. Rare cases of cerebellar involvement have also been described. ${ }^{13}$ Intrafamilial phenotypic variability is present, and families have been described in which some male members have ALD and others are afflicted with other clinical variants. About two-thirds of male patients have overt or subclinical adrenal insufficiency, and a significant proportion have associated gonadal dysfunction. ${ }^{14}$ As many as $50 \%$ of female heterozygotes also have neurological symptoms, and up to $80 \%$ have elevated VLCFA levels. ${ }^{15}$ Neurological symptoms in female heterozygotes usually present later, are milder than those in males and consist of mild pyramidal signs and urinary incontinence. Up to $20 \%$ of females may report severe symptoms resembling AMN. Adrenal and gonadal dysfunction is usually not present in these females, and their condition may be misdiagnosed as multiple sclerosis if a detailed family history is not taken. ${ }^{16}$

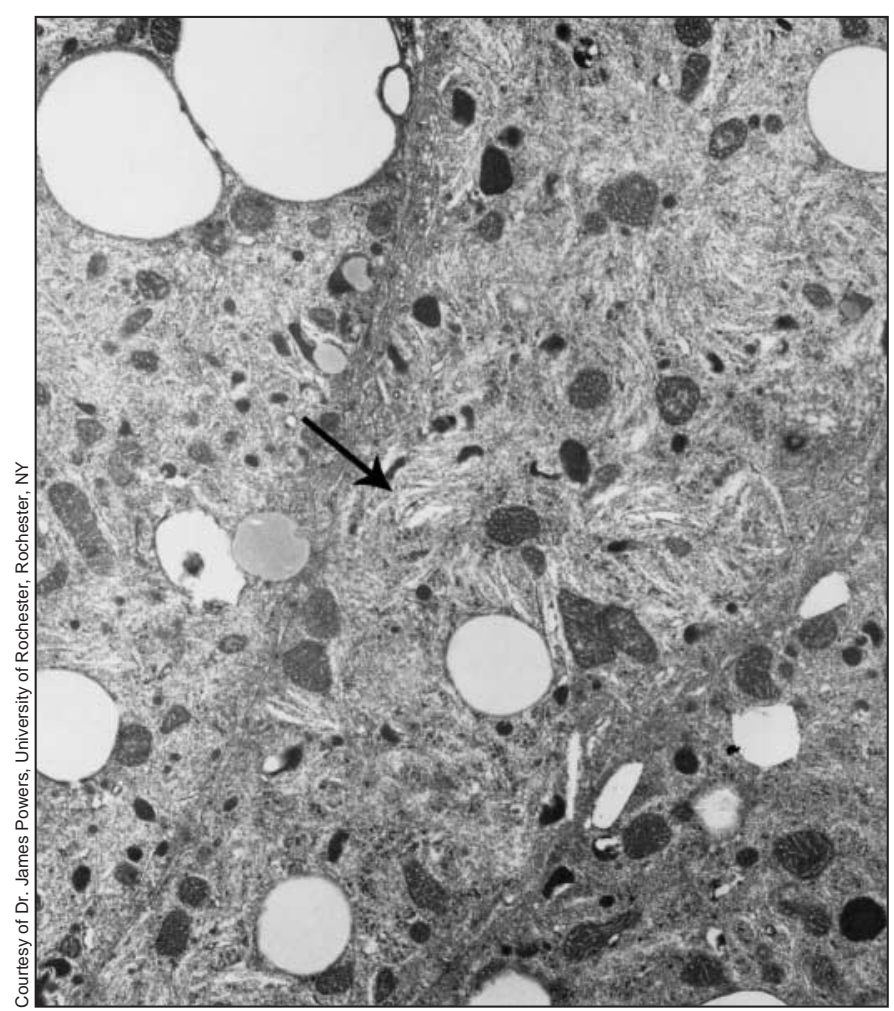

Fig. 2: Electron micrograph of adrenocortical cell of a young boy with adrenoleukodystrophy, illustrating cytoplasmic inclusions of VLCFAs (arrow). Magnification × 35000 .

\section{Diagnosis}

The diagnosis of ALD and AMN is established by measurement of absolute levels of $\mathrm{C}_{26: 0}$ as well as calculation of the $\mathrm{C}_{24: 0} / \mathrm{C}_{22: 0}$ and $\mathrm{C}_{26: 0} / \mathrm{C}_{22: 0}$ ratios. ${ }^{17}$ Rarely, affected males may have normal levels of $\mathrm{C}_{26: 0}$, but all have abnormal ratios. There is no association between the absolute VLCFA levels and the degree of adrenal or neurological involvement. The adrenal insufficiency may either predate or follow the neurological symptoms. Measurement of cortisol and ACTH levels, ACTH stimulation test, and measurement of testosterone and gonadotropin levels may help in establishing the diagnosis of adrenal insufficiency and primary hypogonadism, respectively. An MRI may show evidence of demyelination, and abnormalities in brain stem auditory-evoked potentials, somatosensory-evoked potentials, visual-evoked potentials and motor and sensory nerve conduction velocities may be found..$^{18}$ DNA linkage analysis and mutational analysis are also used. Prenatal diagnosis, with measurement of VLCFA levels in cultured amniocytes and chorion villus cells as well as DNA analysis, is also possible. ${ }^{19}$

Studies have shown that up to $35 \%$ of patients with presumed idiopathic adrenal insufficiency have elevated VLCFA levels and may later experience the associated neurological symptoms. ${ }^{20-22}$ These studies highlight the importance of measuring VLCFA levels in all male patients presumed to have idiopathic adrenal insufficiency; early identification may allow the opportunity for interventions to delay the subsequent onset of neurological symptoms and the opportunity for early identification of asymptomatic family members through screening. Males with "idiopathic" primary adrenal insufficiency should be evaluated for underlying ALD. In addition, males and females presenting with an unknown progressive neurological disorder involving the brain or the spinal cord should be screened for ALD. Measurement of plasma VLCFA levels is the initial screening test of choice; this is followed by genetic analysis. The presence of concomitant adrenal and gonadal dysfunction should be assessed by performing the ACTH stimula-

\begin{tabular}{lccl}
\hline Table 2: Variants of X-linked ALD & \\
\hline & $\begin{array}{c}\text { Frequency } \\
\text { of total, } \%\end{array}$ & $\begin{array}{c}\text { Age at onset of } \\
\text { neurological } \\
\text { symptoms, yr }\end{array}$ & $\begin{array}{c}\text { Main site of } \\
\text { neurological } \\
\text { disease }\end{array}$ \\
$\begin{array}{l}\text { Variant } \\
\text { Childhood cerebral } \\
\text { or classic ALD }\end{array}$ & 45 & 7.1 (SD 1.7) & Cerebrum \\
$\begin{array}{l}\text { Adolescent cerebral } \\
\text { ALD }\end{array}$ & 5 & $10-21$ & Cerebrum \\
$\begin{array}{l}\text { Adult cerebral ALD } \\
\text { AMN }\end{array}$ & 5 & $>21$ & $\begin{array}{l}\text { Cerebrum } \\
\text { Adrenal } \\
\text { insufficiency only }\end{array}$ \\
$\begin{array}{l}\text { Asymptomatic ALD } \\
\text { or AMN }\end{array}$ & 10 & 27 (SD 8) & Spinal cord \\
\hline
\end{tabular}

Note: $\mathrm{SD}=$ standard deviation. 
tion test and by measuring the ACTH, cortisol, testosterone, luteinizing hormone and follicle-stimulating hormone levels.

\section{Management}

The management of patients with AMN is limited and includes hormonal replacement therapy for concomitant adrenal insufficiency or hypogonadism, or both. Therapeutic approaches to arrest or slow the progression of neurological dysfunction have largely been unsuccessful. ${ }^{23}$ The dietary goals are to decrease the exogenous sources of VLCFA by instituting a low-fat diet; however, dietary modifications have not been consistently shown to prevent or slow the course of the disease. Administration of monounsaturated fatty acids leads to competitive inhibition between saturated and unsaturated fatty acid precursors in the microsomal elongation pathway, thereby leading to a reduction in endogenous VLCFA synthesis. ${ }^{24,25}$ A study involving patients with ALD using the combination of dietary restriction and supplementation with glyceryl trioleate and glyceryl trierucate (Lorenzo's oil) produced significant reductions in plasma VLCFA levels but failed to convincingly change the neurological status of the patients. ${ }^{26}$ However, there was a suggestion that treatment with Lorenzo's oil may be helpful in delaying or preventing the onset in asymptomatic but genetically predisposed individuals. ${ }^{27}$ The main side effect associated with glyceryl trierucate therapy is thrombocytopenia, which occurs in up to $40 \%$ of patients. Given the lack of consistent benefit and the risks associated with therapy, it is recommended that such therapy be instituted under the aegis of a supervised research protocol. Bone marrow transplantation, in which the transplanted cells are hypothesized to provide the missing acylCoA synthetase enzyme, has also been evaluated as a therapeutic option. Although bone marrow transplantation has been shown to worsen the neurological status of patients with established severe disease, ${ }^{28}$ it has been found to delay progression in patients with minimal disability. ${ }^{29}$ Other experimental treatments include the use of $\mathrm{HMG}-\mathrm{CoA}$ (3hydroxy-3-methylglutaryl-CoA) reductase inhibitors, cobratoxin, clofibrate, plasmapharesis, glucocorticoids, immunosuppressive agents and pentoxyfilline. ${ }^{30-34}$ These treatments generally have not demonstrated significant benefits.

\section{Summary}

$\mathrm{AMN}$ is a rare but important cause of primary adrenal insufficiency and spastic paraparesis. Although the neurological manifestations are relentlessly progressive, identification and treatment of adrenal dysfunction is life-saving. Our case highlights the importance of screening male patients with presumed idiopathic adrenal insufficiency or spastic paraparesis for underlying AMN or ALD so that the proper diagnosis can be established and genetic counselling provided for the patients and their families. With the evidence that treatment with Lorenzo's oil or bone marrow transplantation, or both, may delay progression of neurological dysfunction in patients with adrenal insufficiency and minimal neurological symptoms and in genetically predisposed family members, early screening and appropriate referral of these individuals assumes an even more important role. ALD and AMN should also be considered in female patients with a neurological disorder of unknown cause involving the brain or spinal cord.

This article has been peer reviewed.

All authors are with the Department of Medicine, University of Saskatchewan, Saskatoon, Sask.

Competing interests: None declared.

Contributors: Stan Van Uum was involved in the initial consideration of the diagnosis of Addison's disease in this patient and in ordering tests to confirm the diagnosis; he also critically reviewed the article for intellectual content and approved the version submitted for publication. Hasnain Khandwala was the attending endocrinologist responsible for analysis and interpretation of the data leading to the the diagnosis of adrenomyeloneuropathy; he reviewed the article for intellectual content, revised the article and approved the version submitted for publication. Regina Taylor-Gjevre was the attending physician at the time of the patient's admission to hospital and assisted wth the preparation of the case report. Monika Spurek was the junior internal medicine resident on-call who was the first to assess, interview, examine and manage this patient in the emergency department; she collected the data for the case report and prepared the final version of the article.

Acknowledgements: We thank Dr. James M. Powers, Professor of Pathology and Neurology, University of Rochester, Rochester, NY, for providing us with the photomicrograph of cytoplasmic inclusions.

\section{References}

1. Van Geel BM, Assies J, Wanders RJA, Barth PG. X-linked adrenoleukodystrophy: clinical presentation, diagnosis and therapy. 7 Neurol Neurosurg Psychiatry $1997 \mathrm{Jul} ; 63(1): 4-14$.

2. Bezman L, Moser AB, Raymond GV, Rinaldo P, Watkins PA, Smith KD, et al. Adrenoleukodystrophy: incidence, new mutation rate, and results of extended family screening. Ann Neurol 2001; 49:512-7.

3. Wanders RJA, Tager JA. Peroxisomal Fatty acid B-oxidation in adrenoleukodystrophy. Dev Neurosci 1991; 13:262-6.

4. Lazo D, Contreras M, Hashmi M, Stanley W, Irazu C, Singh T. Peroxisomal lignoceroyl CoA ligase deficiency in childhood adrenoleukodystrophy and adrenomyeloneuropathy. Proc Natl Acad Sci USA 1988;85:7647-51.

5. Johnson AB, Schaumburg HH, Powers JM. Histochemical characteristics of the striated inclusions of adrenoleukodystrophy. 7 Histochem Cytochem 1976; 24:725-30.

6. Siemerling E, Creudzfeldt HG. Brozzkrankheit und sclerosierende encephalomyelitis. Arch fr psychiatrie 1923;68:217-44.

7. Griffen JW, Goren E, Schaumburg H, Engel WK, Loriaux L. Adrenomyeloneuropathy: a possible variant of adrenoleukodystrophy. Neurol 1977;27 1107-13.

8. Budka H, Sluga E, Heiss WD. Spastic paraplegia associated with Addison's disease: a variant of adrenoleukodystrophy. 7 Neurol 1976;213:237-50.

9. Migeon BR, Moser HW, Moser AB, Axelman J, Sillenece D, Norum RA. Adrenoleukodystrophy: evidence for X linkage, inactivation, and selection favoring the mutant allele in the heterozygous cells. Proc Natl Acad Sci USA 1982;78:5066-70.

10. Ligtenberg MJ, Kemp S, Sarde CO, van Geel BM, Kleijer WJ, Barth PG, et al. Spectrum of mutations in the gene encoding the adrenoleukodystrophy protein. Am 7 Hum Genet 1995;56:44-50.

11. Moser HW, Moser AB, Naidu S, Bergin A. Clinical aspects of adrenoleukodystrophy and adrenomyeloneuropathy. Dev Neurosci 1991;13:254-61.

12. Van Geel BM, Assies J, Weverling GJ, Barth PG. Predominance of the adrenomyeloneuropathy phenotype of $\mathrm{X}$-linked adrenoleukodystrophy in the Netherlands. Neurol 1994;44:2243-6.

13. Ohno T, Tsuchida H, Fukuhara N, Yuasa T, Harayama H, Tsjui S, et al Adrenoleukodystrophy: a clinical variant presenting as olivopontocerebellar atrophy. 7 Neurol 1984;231:167-9.

14. Powers JM, Schaumburg HH. The testis in adrenoleukodystrophy. Am $\mathcal{f}$ Pathol 1981;102:90-8. 
15. Moser AB, Kreiter N, Bezman L, Lu S, Raymond GV, Naidu S, et al. Plasma very long chain fatty acids in 3000 peroxisome disease patients and 29000 controls. Ann Neurol 1999; 45:100-10.

16. Dooley JM, Wright BA. Adrenoleukodystrophy mimicking multiple sclerosis. 7 Can Sci Neurol 1985;12:73-4.

17. Moser HW, Bergin A, Naidu S, Ladenson PW. Adrenoleukodystrophy. Endocrinol Metab Clin North Am 1991;20:297-318.

18. Aubourg P, Adamsbaum C, Lavallard-Rousseau MC, Lamaitre A, Boureau F, Mayer M, et al. Brain MRI and electrophysiologic abnormalities in preclinical and clinical adrenomyeloneuropathy. Neurol 1992;42:85-91.

19. Boue J, Oberle I, Mandel JL, Moser HW, Larsen JW, Dumez Y, et al. First trimester prenatal diagnosis of adrenoleukodystrophy by determination of very long chain fatty acid levels and by linkage analysis to a DNA probe. 7 Hum Genet 1985;69:272-4.

20. Sadeghi-Nejad A, Senior B. Adrenomyeloneuropathy presenting as Addison's disease in childhood. New Engl 7 Med 1990;322:13-6.

21. Lauretti S, Casucci G, Santeusanio F, Angeletti G, Aubourg P, Brunetti P. Xlinked adrenoleukodystrophy is a frequent cause of idiopathic Addison's disease in young adult male patients. 7 Clin Endocrinol Metab 1996;81:470-4.

22. Holmberg BH, Hagg E, Duchek M, Hagenfeldt L. Screening of patients with heredity spastic paraparesis and Addison's disease for adrenoleukodystrophy/adrenomyeloneuropathy. Acta Neurol Scand 1992;85:147-9.

23. Moser HW, Aoubourg P, Cornblath D. Therapy of X-linked adrenoleukodystrophy. In: Desnick RJ, ed. Treatment of genetic diseases. New York: Churchill Livingstone; 1991. p. 111-29.

24. Rizzo WB, Phillips MW, Damman AL. Adrenoleukodystrophy:dietary oleic acid lowers hexacosanoate levels. Ann Neurol 1987;21:232-9.

25. Brown FR, Van Duyn MA, Moser AB. Adrenoleukodystrophy: effects of dietary restriction of very long chain fatty acids and of administration of carnitine and clofibrate on clinical status and plasma fatty acids. Fohns Hopkins Med 7 1982:151;164-72.

26. Aubourg PK, Adamsbaum C, Lavallard-Rousseau MC, Rocchiccioli F, Cartier N, Jambaque I, et al. A two-year trial of oleic and erucic acids ("Lorenzo's oil") as treatment for adrenomyeloneuropathy. New Engl 7 Med 1993;329: 745-52.

27. Uziel G, Bertini E, Bardelli P, Rimoldi M, Gambetti M. Experience on therapy of adrenoleukodystrophy and adrenomyeloneuropathy. Dev Neurosci 1991;13;274-9.

28. Weinberg K, Moser A, Watkins P, Lenarsky C, Winter S, Moser H, et al. Bone marrow transplantation for adrenoleukodystrophy [abstract]. Pediatr Res 1988;23:334A.

29. Aubourg P, Blance S, Jahnbaque I, Kalifa G. Reversible of early neurologic and neuroradiologic manifestations of $\mathrm{X}$-linked adrenoleukodystrophy by bone marrow transplantation. New Engl 7 Med 1990;322:1860-6.

30. Stumpf DA, Hayward A, Haas R, Schuamburg HH. Adrenoleukodystrophy. Failure of immunosuppression to prevent neurologic progression. Arch Neurol 1991;38:48-9.

31. Naidu S, Bresnan MJ, Griffen D, O'toole S, Moser HW. Intensive immunosuppression fails to alter neurological progression in childhood adrenoleukodystrophy. Arch Neurol 1988;45:846-8.

32. Miike T, Taku K, Tamura T, Ohta J, Ozaki MY, Sakai T, et al. Clinical improvement of adrenoleukodystrophy following intravenous gammaglobulin therapy. Brain Dev 1989;11:134-7.

33. Noel P, Nelson S, Bokulic R, Bagby G, Lippton H, Lipscomb G, et al. Pentoxyfilline inhibits lipopolysaccharide-induced serum tumor necrosis factor and mortality. Life Sci 1990;47:1023-9.

34. Singh I, Khan M, Key L, Pai S. Lovastatin for X linked adrenoleukodystrophy. NEngl 7 Med 1998;309:702-3.

Correspondence to: Dr. Hasnain M. Khandwala, Division of Endocrinology, Rm. 3654, Royal University Hospital, 103 Hospital Dr., Saskatoon SK S7N OW8; fax 306 966-7926. hasnain.khandwala@saskatoonhealthregion.ca

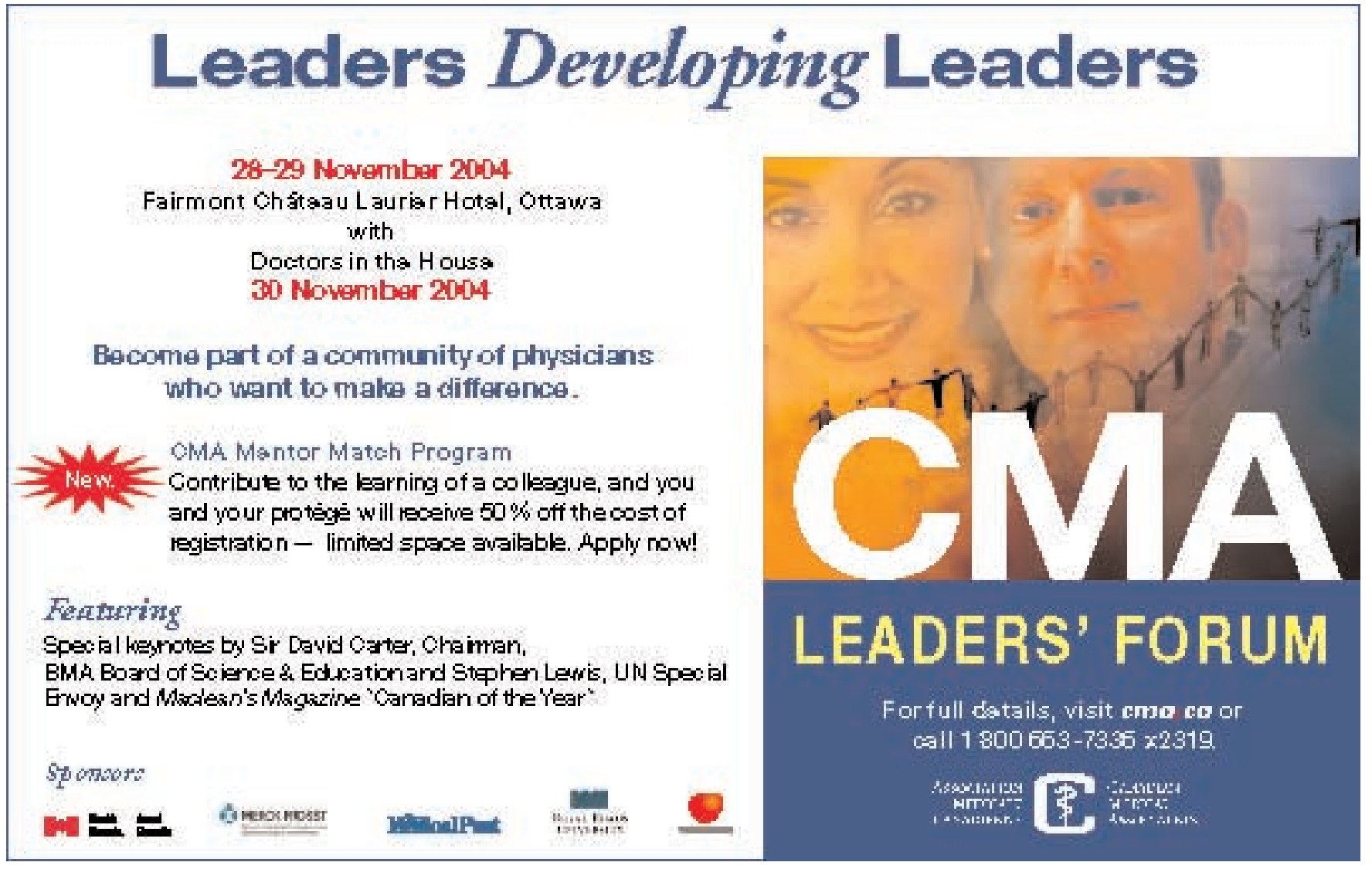

